\title{
Stability of Slotted ALOHA with Spatial Diversity
}

\author{
Vidyut Naware and Lang Tong \\ School of Electrical and Computer Engineering \\ Cornell University, Ithaca, NY 14853. \\ Email :- \{vidyut, ltong\}@ece.cornell.edu
}

\begin{abstract}
We consider the problem of stability of slotted ALOHA for a system consisting of $N$ users communicating with a common receiver, which employs spatial diversity to receive multiple transmissions simultaneuosly. We introduce a general packet reception model to incorporate multiple packet receptions since the collision channel model is no longer valid in such a scenario. We characterize the stability region of slotted ALOHA for the two user case explicitly. We also provide a sufficient condition for stability of slotted ALOHA for the $N>2$ case. Finally, we apply our results to a simple system in which two users communicate with a base station equipped with a linear antenna array. Using stability region as a performance measure, we compare four different receivers/beamformers viz. Matched Filter, Zero Forcing, pseudo-MMSE and true MMSE employed by the base station to receive information from the users.
\end{abstract}

\section{INTRODUCTION}

The recent surge of interest in multiple antenna wireless systems has once again brought into focus the ability of the physical layer to utilize spatial diversity for increasing capacity. Most of the effort in utilizing the spatial diversity at the Media Access Control (MAC) layer has been directed either towards modifying existing protocols like ALOHA, CSMA etc. [10] -[15] or design of new MAC protocols to exploit directional antennas and smart adaptive antenna arrays [16][20]. In systems with spatial diversity, the main issues that need to be addressed are the design of signal processing techniques for the multiple antenna receiver at the physical layer and keeping buffers of packets on the transmitter side under control at the MAC layer. One of the most significant characteristics of such systems is the possibility of receiving information from more than one user simultaneously. To date, the issues of multiple antenna receiver design and buffer stability have been looked at almost in isolation. Our main goal in this paper is to look at the aforementioned problems jointly i.e., to see how signal processing techniques like beamforming affect buffer stability. In particular, we restrict ourselves to slotted ALOHA as the MAC layer protocol and examine the effect of Multipacket receptions on buffer stability.

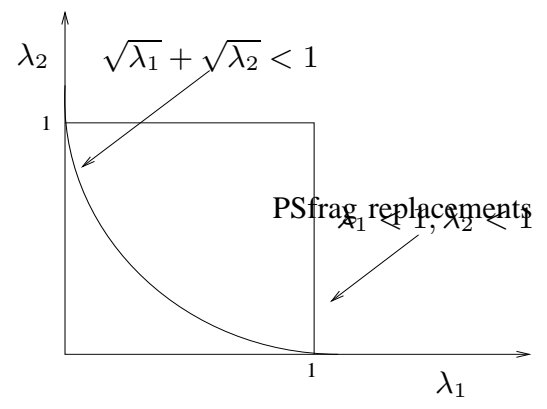

Fig. 1. Two user stability region of slotted ALOHA for the Collision Channel and Orthogonal Channels

\footnotetext{
${ }^{0}$ This work was supported in part by the Office of Naval Research Contract D00014-00-1-0564 and by the Army Research Office Contract ARODAAB19-00-1-0507.
}

In Figure 1, we show the regions of buffer stability i.e., the rate pairs (in terms of packets/slot) for which queues are stable for slotted ALOHA with the collision channel and with perfectly orthogonal channels (no interference). For orthogonal channels we have a unit square, whereas for the collision channel we have a complex form [1]. Our motivation was to look at the behavior of the stability region when the diversity we have lies in between these two extreme cases. We find that the stability region makes a smooth phase transition from concavity to convexity as we move from one extreme to another. In other words, as we allow multipacket receptions to become more likely there comes a point at which the stability region becomes convex. More surprisingly, we find that the stability region is bounded by lines as soon as it becomes convex.

In [7]-[9], a packet radio system is analyzed in which a multiple beam adaptive array is used at the base station to separate users signals. The authors characterize the performance of slotted ALOHA for such a system in terms of the throughput, assuming the users have single packet buffers. In [6], Ghez et al. consider the stability of ALOHA for an infinite user slotted channel with multipacket reception (MPR) capability. In such a channel, the number of packets successfully received in a slot is a random variable which depends only on the number of attempted transmissions in that slot. Thus, this model can capture the event of simultaneous packet successes although it is not sufficient to capture asymmetry among users since all users are treated equal by the model, which need not be true for a multiple antenna wireless system.

Tsybakov and Mikhailov [1] initiated the study of the slotted ALOHA system in terms of the stability of queues at each of the terminals in the system. They found separate necessary and sufficient conditions for stability of the queues in the system using the principle of stochastic dominance. They also found the stability region for the two-user case explicitly. Rao and Ephremides [2] explicitly used the principle of stochastic dominance to find inner bounds to the stability region for the $N>2$ case. Szpankowski [3] found necessary and sufficient conditions for the stability of queues in a slotted ALOHA system for a fixed retransmission probability vector for the $N>2$ case. Recently, Luo and Ephremides [4] introduced the concept of instability ranks in queues to obtain tight inner and outer bounds on the stability region for the $N>2$ case. However, to date there is no closed form characterization of the stability region for the $N>2$ case. The point to note is that the above results were derived assuming the collision channel model for packet success-an assumption that we relax.

The remainder of this paper is organized as follows. In section II, we specify the system model and its operation. In section III, we derive the stability region for the two user case. We also characterize some interesting properties of this region. In section IV, we give sufficient conditions for stability for the $N>2$ case. In section V, we apply our analytical results to four different receiver structures viz., Decorrelating, Matched filter, pseudo-MMSE and true MMSE and compare their performance in terms of the stability region to 
gain some insights. Finally, we conclude in section VI.

\section{System Model}

The system consists of $N$ users, each having an infinite buffer, communicating with a common receiver. The receiver has multiple antennas used to implement beamforming for receiving multiple packets simultaneously. The channel is slotted in time and a slot duration equals the packet transmission time. Packets are assumed to be of equal length for all the users. The arrivals at the $i$ th queue $(i \in\{1,2, \ldots, N\})$ are independant and identically distributed Bernoulli random variables from slot to slot with mean $\lambda_{i}$. Arrival processes are assumed to be independant from user to user. If the $i$ th users' buffer is nonempty, he transmits a packet with probability $p_{i}$ in a slot.

Now we define a very general packet reception model to capture the event of multipacket reception. Suppose that the set $\mathcal{S} \subseteq$ $\{1,2, \ldots, N\}$ of users transmit in a slot, then we define for $i \in \mathcal{S}$,

$$
\begin{aligned}
& q_{i \mid \mathcal{S}}=\operatorname{Pr}\{i \text { th users' packet is successfully } \\
& \text { received } \mid \mathcal{S} \text { transmits }\}
\end{aligned}
$$

We assume that user $i$ 's packet is successfully received independantly from slot to slot. We assume that a perfect feedback mechanism exists so that users retain packets which did not get through. It should be clear that the conditional probabilities $q_{i \mid \mathcal{S}}$ are a function of the receiver front-end which will be employed by the receiver to "separate" users' signals. Before we proceed to derive some of the results of the next section, a few definitions are in order. We use the definition of stability used by Loynes [5].

Definition: A multidimensional stochastic process, $\mathbf{Q}^{t}=$ $\left(Q_{1}^{t}, \ldots, Q_{N}^{t}\right)$ is stable if for $\mathbf{x} \in \mathbb{N}^{N}$ the following holds

$$
\lim _{t \rightarrow \infty} \operatorname{Pr}\left\{\mathbf{Q}^{t}<\mathbf{x}\right\}=F(\mathbf{x}) \text { and } \lim _{\mathbf{x} \rightarrow \infty} F(\mathbf{x})=1 .
$$

If a weaker condition holds viz.,

$$
\lim _{\mathbf{x} \rightarrow \infty} \liminf _{t \rightarrow \infty} \operatorname{Pr}\left\{\mathbf{Q}^{t}<\mathbf{x}\right\}=1
$$

then the process is called substable. Further, the process is said to be unstable if it is not substable.

It can be easily shown that stability implies substability. For the slotted ALOHA system we described in Section II, the stochastic process under consideration is the queue length at the $N$ buffers. Thus, $Q_{i}^{t}$ represents the queue length at $i$ th buffer at time $t$. Because of the special arrival and departure statistics in this system, the $N$ dimensional queue evolution is an aperiodic and irreducible Markov chain. The notion of stability in this system is then equivalent to the positive recurrence of the Markov chain. Intuitively, stability means that the buffers in the system are not growing to infinity.

Definition: For an $N$ user slotted ALOHA buffer system, the stability region is defined as the set of arrival rates $\lambda=\left[\lambda_{1}, \lambda_{2}, \ldots, \lambda_{N}\right]$ for which there exists a retransmission probability vector $\mathbf{p}=$ $\left[p_{1}, p_{2}, \ldots, p_{N}\right]$ such that the buffers in the system are stable. The stability region clearly depends on the underlying packet reception model.

\section{StabiLity REgION FOR THE TWO USER CASE}

Since the stability region for the collision channel is unknown for the $N>2$ case, we first find the stability region for the $N=2$ case for the general reception model given by (1). For notational

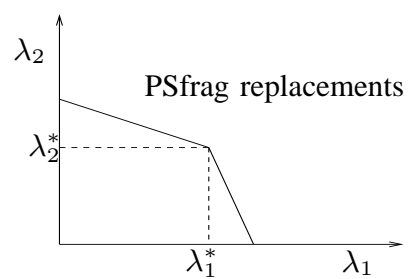

Fig. 2. Stability region for a fixed retransmission probability vector $\left[p_{1}, p_{2}\right]$

convenience, we define the probabilities of packet success in the two user case as

$$
\begin{aligned}
& q_{i}^{(1)}=\operatorname{Pr}\{\text { user } i \text { is successful } \mid \text { only user } i \text { transmits }\} \\
& q_{i}^{(2)}=\operatorname{Pr}\{\text { only user } i \text { is successful } \mid \text { both users transmit }\} \\
& q^{(2)}=\operatorname{Pr}\{\text { both users are successful } \mid \text { both users transmit }\}
\end{aligned}
$$

Further, we define $Q_{1} \triangleq q_{1}^{(1)}-q_{1}^{(2)}-q^{(2)}$ and $Q_{2} \triangleq q_{2}^{(1)}-$ $q_{2}^{(2)}-q^{(2)}$. Thus, $Q_{1}$ and $Q_{2}$ denote the difference between the (conditional) probability of success in the absence of interference and the (conditional) probability of success in the presence of interference for the users. Note that the above probabilities can capture not only all possible packet reception events but also correlations among those events and user asymmetry. To find the stability region, we first need to find the stability region of the system for a fixed retransmission probability vector $\mathbf{p}\left(=\left[p_{1}, p_{2}\right]\right)$. The following lemma gives us exactly that.

Lemma 1: If $Q_{1} \geq 0$ and $Q_{2} \geq 0$, the stability region of slotted ALOHA for the general packet reception model for a given $\left[p_{1}, p_{2}\right]$ is given by

$$
\lambda_{1}<p_{1} q_{1}^{(1)}-\frac{p_{1} p_{2} \lambda_{2} Q_{1}}{\lambda_{2}^{*}}, \text { for } \lambda_{2}<\lambda_{2}^{*}
$$

and

$$
\lambda_{2}<p_{2} q_{2}^{(1)}-\frac{p_{1} p_{2} \lambda_{1} Q_{2}}{\lambda_{1}^{*}}, \text { for } \lambda_{1}<\lambda_{1}^{*}
$$

where,

$$
\lambda_{1}^{*}=p_{1} q_{1}^{(1)}-p_{1} p_{2} Q_{1} \text { and } \lambda_{2}^{*}=p_{2} q_{2}^{(1)}-p_{1} p_{2} Q_{2}
$$

Proof: We use the idea of stochastic dominance and use an argument similar to that by Rao and Ephremides [2].

Figure 2 shows us the stability region as given by Lemma 1 . The conditions $Q_{1} \geq 0$ and $Q_{2} \geq 0$ are needed for the stochastic dominance of the associated dominant systems. In fact, these conditions are equivalent to the probability of success of any user in the presence of interference (from the other user) be no greater than the probability of success in the absence of interference-a reasonable and practical assumption.

We now give a key result of this paper in the form of this theorem.

Theorem 1: If $Q_{1} \geq 0$ and $Q_{2} \geq 0$, then the stability region of slotted ALOHA for the general reception model is given by $\mathcal{R}_{1} \cap \mathcal{R}_{2}$ where

$$
\begin{gathered}
\mathcal{R}_{1} \triangleq\left\{\left(\lambda_{1}, \lambda_{2}\right):\left(\lambda_{1}, \lambda_{2}\right) \geq(0,0),\left(\lambda_{1}, \lambda_{2}\right)\right. \text { lies } \\
\text { below the curve } \left.\lambda_{2}=f\left(\lambda_{1}, q_{1}^{(1)}, q_{2}^{(1)}, Q_{1}, Q_{2}\right)\right\}
\end{gathered}
$$

and

$$
\begin{gathered}
\mathcal{R}_{2} \triangleq\left\{\left(\lambda_{1}, \lambda_{2}\right):\left(\lambda_{1}, \lambda_{2}\right) \geq(0,0),\left(\lambda_{1}, \lambda_{2}\right)\right. \text { lies } \\
\text { below the curve } \left.\lambda_{1}=f\left(\lambda_{2}, q_{2}^{(1)}, q_{1}^{(1)}, Q_{2}, Q_{1}\right)\right\}
\end{gathered}
$$


where

$$
f(\lambda, \alpha, \beta, \gamma, \delta)= \begin{cases}\beta-\frac{\lambda \delta}{\alpha-\gamma}, & \lambda \in \mathcal{I}_{1} \\ \frac{(\sqrt{\alpha \beta}-\sqrt{\lambda \delta})^{2}}{\gamma}, & \lambda \in \mathcal{I}_{2}\end{cases}
$$

where

$$
\mathcal{I}_{1}=\left[0, \frac{\beta(\alpha-\gamma)^{2}}{\alpha \delta}\right] \text { and } \mathcal{I}_{2}=\left(\frac{\beta(\alpha-\gamma)^{2}}{\alpha \delta}, \frac{\alpha \beta}{\delta}\right] .
$$

If either $Q_{1}$ or $Q_{2}$ equals zero, then we assume $\frac{1}{0}=\infty$ and our result still holds.

Proof: We use Lemma 1. Since we know the stability region for a fixed retransmission probability vector $\mathbf{p}$, we need to find the union of all the stability regions as the parameter $\mathbf{p}$ varies over $[0,1]^{2}$. One way of doing this is to setup a corresponding constrained optimization problem i.e. for a fixed $\lambda_{1}$, maximize $\lambda_{2}$ as $\mathbf{p}$ varies over $[0,1]^{2}$, where $\lambda_{1}$ and $\lambda_{2}$ are related by (4) and (5). This is the method which we used in our proof [22].

We note a few interesting things about the stability region. First, the function $f$ characterizing the stability region in (8) is linear for some part of the domain and is strictly convex in the remainder of its domain. The stability region for the two user collision channel can be found as a special case of our model with $q_{1}^{(1)}=1, q_{2}^{(1)}=1, q_{1}^{(2)}=$ $0, q_{2}^{(2)}=0$ and $q^{(2)}=0$ and is bounded by the curve $\sqrt{\lambda_{1}}+\sqrt{\lambda_{2}}=$ 1 , which is strictly convex everywhere. In fact, it is easy to see from (8) that the interval where $f$ is linear has non-zero Lebesgue measure as soon as there is a nonzero probability of success in the presence of interference i.e. $q_{1}^{(1)}-Q_{1}>0$. Thus, there is a characteristic change in the structure of the stability region as soon as we have multipacket reception. Second, we see that there is a symmetry in the way the two regions $\mathcal{R}_{1}$ and $\mathcal{R}_{2}$ are defined in terms of the function $f$. Third, the stability region is entirely characterized by $q_{1}^{(1)}, q_{2}^{(1)}, Q_{1}$ and $Q_{2}$. In turn, $Q_{1}$ and $Q_{2}$ depend only on the marginal probabilities of success in the presence and absence of interference, which is not surprising since the two users are not collaborating their packet transmissions.

Further, we observe the following three important properties of the stability region:

Property 1: (P1) - Assume that $q_{1}^{(1)}>0, q_{2}^{(1)}>0, q_{1}^{(1)}-Q_{1}>0$ and $q_{2}^{(1)}-Q_{2}>0$ i.e. non-zero probability of success in the presence and absence of interference. Then, the stability region is bounded by lines iff

$$
\frac{Q_{1}}{q_{1}^{(1)}}+\frac{Q_{2}}{q_{2}^{(1)}} \leq 1
$$

Property 2: (P2) - Assume that $q_{1}^{(1)}>0, q_{2}^{(1)}>0, q_{1}^{(1)}-Q_{1}>0$ and $q_{2}^{(1)}-Q_{2}>0$. Then, the stability region is bounded by lines iff the stability region is convex.

Property 3: (P3) - If the stability region is not bounded by lines, then the strictly convex parts of $f$ which bound the regions $\mathcal{R}_{1}$ and $\mathcal{R}_{2}$ coincide on the boundary of the stability region.

Figure 3 shows the stability regions characterized by the $\mathbf{q}$ vector based on the above properties. P1 has a nice interpretation; there is a critical point for the $\mathbf{q}$ vector at which the behavior of the stability region makes a phase transition from a complex form to a simple form (bounded by lines). Further, this critical point depends only on the sum of the ratios of probability of success of users in the presence of interference to that in the absence of interference.

P2 tells us that the two simple properties (convexity and being bounded by lines) of a region are equivalent for the stability region. The condition of the stability region being bounded by lines and being convex corresponds to a regime in which when one user increases

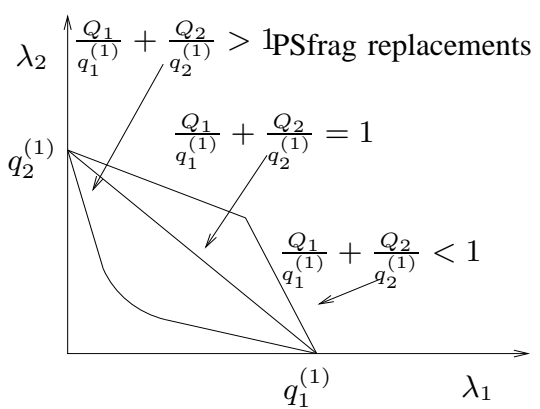

Fig. 3. The shape of the stability region for different $\mathbf{q}$ vectors with $q_{1}^{(1)}$ and $q_{2}^{(1)}$ fixed.

his rate, the other users' maximum supportable rate decreases only linearly, and that too at a rate which is low till a certain point and then suddenly increases. Another interpretation is that when the stability region is convex then higher sum rates can be achieved. In addition, when the stability region is convex we know that if two rate pairs are stable then any rate pair lying on the line segment joining those two rate pairs is also stable. This is an important point because the stability region for the two user collision channel is not convex. When equality holds in equation (10), the stability region is a triangle as shown in Figure 3. All the rate pairs in this region can be stabilized by TDMA schemes (even in a collision channel). Thus, the condition $\frac{Q_{1}}{q_{1}^{(1)}}+\frac{Q_{2}}{q_{2}^{(1)}}<1$ gives us the regime in which a distributed strategy like slotted ALOHA can do better than a centralized TDMA scheme.

\section{BOUNDS FOR THE $N>2$ CASE}

Till this point we have considered the stability region for the two user case. Finding the stability region for the $N>2$ case is harder since the number of the parameters required to define the general reception model increases rapidly in addition to more complex queue interactions. So in this section, we give a sufficient condition for stability for $N>2$ for the reception model given by (1).

Lemma 2: Let $\mathcal{N} \triangleq\{1,2, \ldots, N\}$. If $\forall l \in \mathcal{N}, \forall \mathcal{A} \subseteq \mathcal{N} \backslash\{l\}$ and $\forall \mathbf{p}=\left[p_{1}, p_{2}, \ldots, p_{N}\right] \in[0,1]^{N}$,

$$
p_{l} \sum_{\mathcal{S} \subseteq \mathcal{A}}\left(\prod_{i \in \mathcal{S}} p_{i} \prod_{j \in \mathcal{A} \backslash \mathcal{S}} \bar{p}_{j}\right) q_{l \mid(\mathcal{S} \cup\{l\})} \geq a_{l}(\mathbf{p})
$$

where,

$$
a_{l}(\mathbf{p})=p_{l} \sum_{\mathcal{S} \subseteq \mathcal{N} \backslash\{l\}}\left(\prod_{i \in \mathcal{S}} p_{i} \prod_{j \in \mathcal{N} \backslash\{\mathcal{S} \cup\{l\}\}} \bar{p}_{j}\right) q_{l \mid(\mathcal{S} \cup\{l\})}
$$

then, an inner bound of the stability region is given by the region $\mathcal{R}$, where,

$$
\mathcal{R}=\bigcup_{\mathbf{p} \in[0,1]^{N}} \mathcal{R}_{\mathbf{p}}
$$

where $\mathcal{R}_{\mathbf{p}}$ is defined as,

$$
\mathcal{R}_{\mathbf{p}}=\left\{\left(\lambda_{1}, \lambda_{2}, \ldots, \lambda_{N}\right) \geq \mathbf{0}: \lambda_{l}<a_{l}(\mathbf{p}), \forall l \in \mathcal{N}\right\} .
$$

We will not prove this lemma but it follows by application of the concept of stochastic dominance to the $N$ queues [1]. We consider a parallel ALOHA system in which every queue is persistent [3] viz., every queue continues to transmit dummy packets even when it is empty. It can easily be shown that the region $\mathcal{R}$ given by Lemma 2 is the stability region of this parallel dominant system. Using stochastic dominance, we can conclude that even the original system 
is stable for arrival rates inside $\mathcal{R}$. Equation (11) is the condition that the probability of success for any user when all the $N$ buffers are nonempty be atmost equal to the probabilities of success when atleast one buffer is empty - a condition required for stochastic dominance to hold. We have also given a tighter inner bound of the stability region using weaker stochastic dominant systems in [21] albeit for a fixed retransmission probability vector $(\mathbf{p})$.

\section{ReCEIVER PERformance}

To get more insights for the analytical results in section III and as an example, we now apply our results to a two-user cellular scenario to compare different receiver front-ends.

We consider two users, each communicating with a central base station that employs a linear array of $M$ antennas. The two users use slotted ALOHA as the MAC. We assume that the slots are perfectly synchronized. The two users are located relatively far away from the base station at fixed angular postions $\boldsymbol{\theta}=\left[\theta_{1}, \theta_{2}\right]$ with respect to the array normal. We assume that most of the energy from user transmissions is received from a planar wavefront arriving at the angle $\boldsymbol{\theta}$. Under these assumptions, we can describe the received signal at the base station $\mathbf{y}$ as

$$
\mathbf{y}=\mathbf{V}(\boldsymbol{\theta}) \mathbf{H} \mathbf{s}+\mathbf{n}
$$

where $\mathbf{V}(\boldsymbol{\theta})$ is a Vandermonde matrix of array responses, $\mathbf{H}=$ $\operatorname{diag}\left[h_{1}, h_{2}\right]$, is a diagonal matrix of channel (flat) fading for the two users, $\mathbf{s}=\left[s_{1}, s_{2}\right]^{T}$, is a vector of users' transmitted symbols and $\mathbf{n} \sim \mathcal{C N}\left(0, \mathbf{I}_{M}\right)$, is additive white gaussian noise.

We also assume that the channel fading is slow and it is independant for the two users and also is i.i.d. from slot to slot. For our numerical results, we assume Rayleigh fading with zero mean and covariance matrix $\Sigma=\operatorname{diag}\left[\sigma_{h_{1}}^{2}, \sigma_{h_{2}}^{2}\right]$. User symbols $\left(s_{i}, i=1,2\right)$ are independant of each other and the channel fading with $\mathbb{E}\left(\left\|s_{i}\right\|^{2}\right)=1, i=1,2$. We also assume that the base station does not know the channel realization, when it implements the frontend. We represent the front-end processing by $\mathbf{F}$ ( $i$ th row of $\mathbf{F}$ is the set of beamforming weights for the $i$ th user) as follows:

$$
\begin{aligned}
\mathbf{z}=\mathbf{F y} & =\overbrace{\mathbf{F V}(\boldsymbol{\theta}) \mathbf{H}}^{\mathbf{R}} \mathbf{s}+\mathbf{F n} \\
& =\mathbf{R s}+\mathbf{w}
\end{aligned}
$$

The most important assumption we make is that of the SINR threshold model for packet success i.e., a packet is successfully received and decoded for user $i$ if

$$
\mathbb{E}\left(\operatorname{SINR}_{i} \mid \operatorname{channel}(\mathrm{s})\right)=\frac{\left\|r_{i i}\right\|^{2}}{\left\|r_{i \bar{i}}\right\|^{2}+\mathbb{E}\left(\left\|w_{i}\right\|^{2}\right)}>\text { Threshold }=\tau
$$

where $\bar{i}=\{1,2\} \backslash\{i\}$. Under the SINR threshold model, the $\mathbf{q}$ vector of packet success probabilities for a particular $\mathbf{F}$ can be found as

$$
\begin{gathered}
q_{i}^{(1)}=\operatorname{Pr}\left\{\frac{\left\|r_{i i}\right\|^{2}}{\mathbb{E}\left(\left\|w_{i}\right\|^{2}\right)}>\tau\right\} \\
q_{i}^{(2)}=\operatorname{Pr}\left\{\frac{\left\|r_{i i}\right\|^{2}}{\left\|r_{i \bar{i}}\right\|^{2}+\mathbb{E}\left(\left\|w_{i}\right\|^{2}\right)}>\tau, \frac{\left\|r_{\overline{i i}}\right\|^{2}}{\left\|r_{\bar{i} i}\right\|^{2}+\mathbb{E}\left(\left\|w_{\bar{i}}\right\|^{2}\right)} \leq \tau\right\} \\
q^{(2)}=\operatorname{Pr}\left\{\frac{\left\|r_{11}\right\|^{2}}{\left\|r_{12}\right\|^{2}+\mathbb{E}\left(\left\|w_{1}\right\|^{2}\right)}>\tau, \frac{\left\|r_{22}\right\|^{2}}{\left\|r_{21}\right\|^{2}+\mathbb{E}\left(\left\|w_{2}\right\|^{2}\right)}>\tau\right\}
\end{gathered}
$$

We now consider the performance of four different front-ends for the above system.

1) Zero forcing or Decorrelating (ZF): $\mathbf{F}$ is the pseudo-inverse of $\mathbf{V}(\boldsymbol{\theta})$.

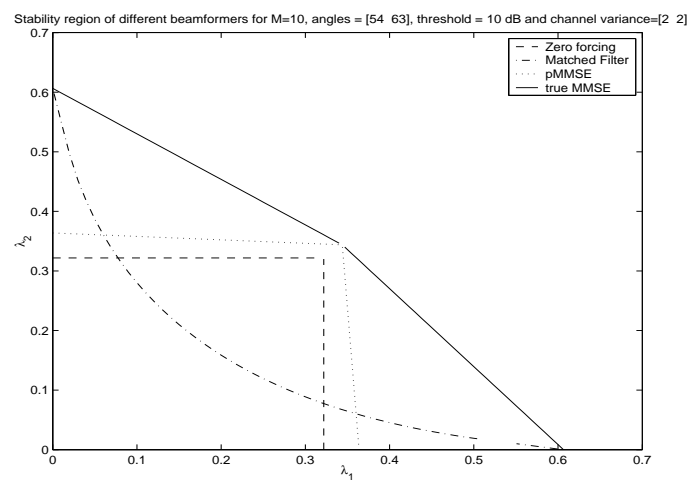

Fig. 4. $M=10, \boldsymbol{\theta}=[54,63]$, Threshold $=10 d B$, channel gain $=3 d B$

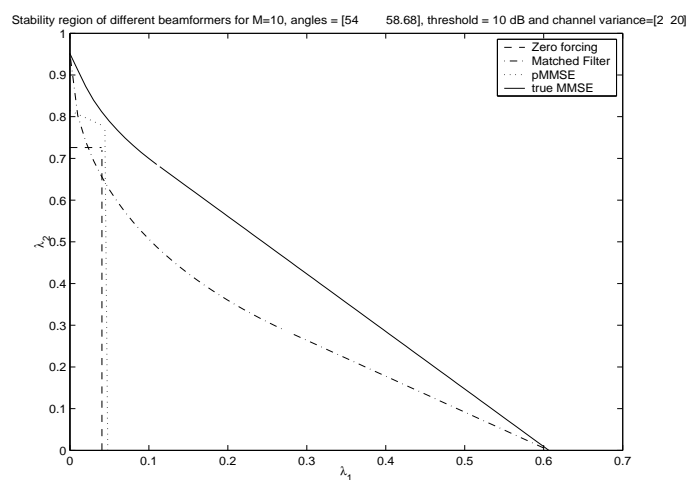

Fig. 5. $M=10, \boldsymbol{\theta}=[54,58]$, Threshold $=10 d B$, channel gains $=$ $[3,13] d B$

2) Matched Filter (MF): $\mathbf{F}=\mathbf{V}^{H}(\boldsymbol{\theta})$.

3) pseudo-MMSE (pMMSE): For this receiver, $\mathbf{F}=$ $\operatorname{diag}\left[\sigma_{h_{1}}^{2}, \sigma_{h_{2}}^{2}\right] \mathbf{V}^{H}(\boldsymbol{\theta}) \mathbf{R}_{y y}^{-1}$, where $\mathbf{R}_{y y}$ is the correlation matrix of $\mathbf{y}$ assuming both users transmit. Note that the perfect MMSE receiver needs to know which users are transmitting in order to find the optimal weights.

4) true-MMSE (MMSE): The true MMSE receiver knows which users are transmitting in a slot and thus adapts its weights optimally. Clearly, when only one user is transmitting it is the same as the MF and when both users are transmitting it is the pMMSE.

Using the stability region as a figure of merit, we can now compare the stability regions of these front-ends in various situations of interest.

\section{A. Symmetric Case}

In this case, the channels for the two users are symmetric i.e. $\sigma_{h_{1}}^{2}=\sigma_{h_{2}}^{2}$. In Figure 4 , we see stability regions for the different front-ends when the two users are relatively close, $\boldsymbol{\theta}=[54,63]^{\circ}$. We observe that in this rather pessimistic scenario, the MF performs better when one of the users demands a very low rate whereas the ZF and pMMSE perform a little better when both users demand an equal rate. We also note that the stability region of the $\mathrm{ZF}$ is a rectangle since, the ZF decouples the two users signals perfectly. Also, it is not surprising that the true MMSE outperforms all the other receivers since it is the optimal receiver. 


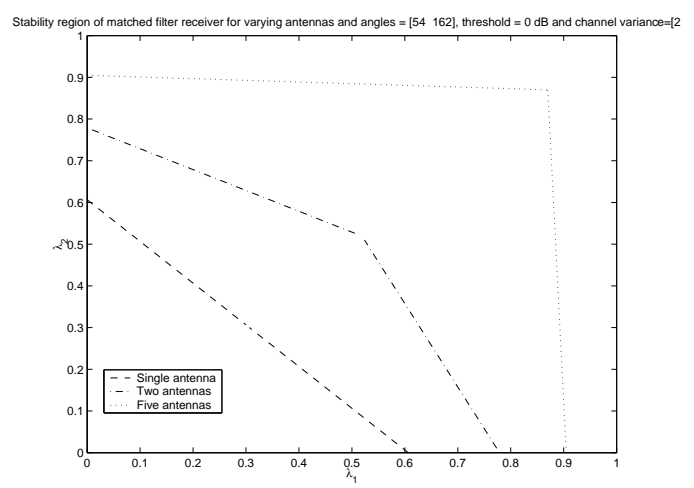

Fig. 6. $\boldsymbol{\theta}=[54,162]$, Threshold $=0 d B$, channel gain $=3 d B$

\section{B. Asymmetric Case}

Figure 5 shows the situation when the second user has a very good channel as compared to the first and the users are almost colinear, $\boldsymbol{\theta}=[54,58]^{\circ}$. We see a near-far effect with the ZF and pMMSE front-ends, whereas the MF performs very well. It is not surprising since the MF does not really try to null out the other user while the $\mathrm{ZF}$ and pMMSE do that. Because of the angular proximity, the ZF and pMMSE suffer. We also note with interest that the stability region of the pMMSE contains the stability region of the ZF receiver in Figures 4 and 5.

\section{Matched Filter, Symmetric Case}

Since the MF performs so well, now we see how varying the number of antenna elements changes the stability region of the MF. In figure 6 , we see that the stability region gets bigger as the number of antenna elements increase, as expected. It also shows how having more antennas allows us to achieve higher rates in the regime where the user rates are equal. In fact, it can be easily deduced that there would be a certain threshold on the number of antenna elements after which, the gain that can be had from using spatial diversity is insignificant.

For $N>2$, we observe that the bound given by Lemma 2 is actually the stability region for the ZF receiver assuming $M>N$ and $\mathbf{V}(\boldsymbol{\theta})$ has full column rank. In this case, the probability of success of any user does not depend on other users' transmission and so the stability region is a $N$ dimensional box, which is identical to the region $\mathcal{R}$ specified by Lemma 2 for the reception model corresponding to the $\mathrm{ZF}$ case.

\section{CONCLUSIONS}

In this paper, we considered the problem of stability of slotted ALOHA for a general reception model intended to capture the behavior of a multiple antenna wireless system. We characterized the stability region of slotted ALOHA for the two user case. We found that in some cases the two user stability region has a simple structure as given by $\mathrm{P} 1$ and $\mathrm{P} 2$. We show that the stability region makes a phase transition from concavity to convexity as the degree of spatial diversity increases. We also found that the stability region is characterized completely by the marginal probabilities of success of the users. We conjecture that the stability region for the $N>2$ user case is bounded by hyperplanes in some multipacket reception regime. In fact, it would be interesting to see if we get some properties analogous to $\mathrm{P} 1, \mathrm{P} 2$ and $\mathrm{P} 3$ for the $N>2$ stability region. In the specific two user scenario that we considered, the performance of the relatively simple Matched filter was notably good-which is quite surprising. Future work would be directed towards generalizing our results to the $N>2$ case. We would also like explore the implications of stability on the statistics of delay in slotted ALOHA based random access systems.

\section{REFERENCES}

[1] B. Tsybakov and W. Mikhailov, "Ergodicity of slotted ALOHA systems, " Probl. Inform. Transmission, vol.15, no. 4, pp. 73-87, 1979.

[2] R. Rao and A. Ephremides, " On the stability of interacting queues in a multi-access system, " IEEE Trans. Inform. Theory, vol. 34, pp. 918-930, Sept. 1988.

[3] W. Szpankowski, "Stability conditions for some mulitqueue distributed systems: Buffered random access systems," Adv. Appl. Probab., vol. 26, pp. 498-515, 1994.

[4] W. Luo and A. Ephremides, "Stability of $N$ interacting queues in randomaccess Systems," IEEE Trans. Inform. Theory, vol. 45, no. 5, pp. 15791587, July 1999.

[5] R. M. Loynes, "The stability of a queue with non-independant inter-arrival and service times," Proc. Cambridge Philos. Soc., vol. 58, pp. 497-520, 1962.

[6] S. Ghez, S. Verdu and S. Schwartz, "Stability properties of slotted ALOHA with multipacket reception capability," IEEE Trans. Automat. Cont., vol. 33, no. 7, pp. 640-649, July 1988.

[7] R. T. Compton, Jr. and J. Ward, "High throughput slotted ALOHA packet radio networks with adaptive arrays ," IEEE Trans. Comm., vol. 41, no. 3, pp. 460-470, March 1993.

[8] C. T. Lau and C. Leung, "A slotted ALOHA packet radio system with multiple antennas and receivers," IEEE Trans. Vehic. Tech., vol. 39, no. 3, pp. 218-226, August 1990 .

[9] P. M. Soni and A. Chockalingam, "Performance analysis od space diversity s-ALOHA with steerable beam smart antenna arrays," Proc. of GLOBECOM, vol. 1, pp. 36-41, 2000.

[10] Y. Ko, V. Shankarkumar and N. H. Vaidya, "Medium access control protocols using directional antennas in ad hoc networks," Proc. of IEEE INFOCOM, pp. 13-21, 2000.

[11] J. Zander, "Slotted ALOHA multihop packet radio networks with directional antennas," Electronics Letters, vol. 26, no. 25, pp. 2098-2099, 2000.

[12] J. M. G. Linnartz, "Slotted ALOHA mobile network with vertical array antenna diversity," Proc. of GLOBECOM, vol. 1, pp. 79-82, 1994.

[13] R. Zhang, M. K. Tsatsanis and N. D. Sidiropoulos, "Performance analysis of a random access packet radio system with joint network-spatial diversity," Proc. of ICASSP, vol. 5, pp. 2593-2596, 2000.

[14] R. Stridh and B. Ottersten, "Packet data throughput for wireless systems with smart antennas," Proc. of VTC, vol. 1, pp. 32-36, 1999.

[15] T. Kobayashi and I. Sasase, "Performance analysis of packet reservation multiple access with an adaptive array," Proc. of PIMRC, vol. 3, pp. 923927, 1997.

[16] M. Horneffer and D. Plassmann, "Directed antennas in the mobile broadband system," Proc. of INFOCOM, vol. 2, pp. 704-712, 1996.

[17] F. Shad, T. D. Todd, V. Kezys and J. Litva, " Dynamic slot allocation (DSA) in indoor SDMA/TDMA using a smart antenna base station," IEEE Trans. Networking, vol. 9, pp. 69-81, 2001.

[18] S. V. Krishnamurthy, A. S. Acampora and M. Zorzi, "Polling-based media access protocols for use with smart adaptive array antennas," IEEE/ACM Trans. Networking, vol. 9, no. 2, pp. 148-161, 2001.

[19] H. Yin and H. Liu, "An SDMA protocol for wireless multimedia networks," Proc. of ICASSP, vol. 5, pp. 2613-2616, 2000.

[20] T. S. Yum and K. W. Hung, "Design algorithms for multihop packet radio networks with multiple directional antennas stations," IEEE Trans. Comm., vol. 40, no. 11, pp. 1716-1724, 1992.

[21] V. Naware and L. Tong, "Stability of Queues in Slotted Aloha with Multiple Antennas," Proc. 40th Annual Allerton Conference on Communication, Control, and Computing, October, 2002.

[22] V. Naware, G. Mergen and L. Tong, "On the stability of slotted ALOHA for systems with spatial diversity," to be submitted to IEEE Trans. Inform. Theory. 